\title{
Questes
}

\section{Les pèlerinages dans le Saint-Empire à la fin du Moyen Âge : mises en perspective}

Jean-Dominique Delle Luche

\section{(2) OpenEdition}

Édition électronique

URL : http://journals.openedition.org/questes/1426

DOI : 10.4000/questes.1426

ISSN : 2109-9472

Éditeur

Les Amis de Questes

\section{Édition imprimée}

Date de publication : 15 novembre 2011

Pagination : 78-92

ISSN : 2102-7188

\section{Référence électronique}

Jean-Dominique Delle Luche, « Les pèlerinages dans le Saint-Empire à la fin du Moyen Âge : mises en perspective », Questes [En ligne], 22 | 2011, mis en ligne le 01 janvier 2014, consulté le 01 mai 2019. URL : http://journals.openedition.org/questes/1426 ; DOI : 10.4000/questes.1426 


\title{
Les pèlerinages dans le Saint-Empire à la fin du Moyen Âge : mises en perspective
}

\author{
Jean-Dominique DELLE LUCHE
}

Évoquer les pèlerinages dans le Saint-Empire à la fin du Moyen Âge, c'est revenir sur un sujet classique de la civilisation médiévale, mais d'une manière singulièrement éloignée de l'image traditionnelle qui associe le plus souvent le pèlerin au long voyage vers Compostelle ou Jérusalem, le situant qui plus est spectaculairement au temps des croisades. Placer l'étude des pèlerinages au Moyen Âge tardif permet en effet de sortir de ce stéréotype et de leur donner un nouveau sens dans un contexte historique et historiographique différent, à la croisée d'un réseau de questions : y a-t-il moins d'inconnues et de périls pour le voyageur en l'an 1500 qu'en l'an 1100 ? Quel rôle joue désormais dans la carte des pèlerinages «l'autre Europe » catholique qui a émergé au cours des siècles centraux du Moyen Âge, autour d'États pérennes comme la Pologne, la Bohême, la Hongrie, les États scandinaves ainsi que les espaces de la Hanse et de l'Ordre teutonique ? Quel a été l'impact de la perte définitive de Jérusalem et du déclin des États chrétiens de l'Est du bassin méditerranéen sur les routes de pèlerinages et la manière de voyager, maintenant que la croisade est vouée à l'échec sans que l'on abandonne toutefois cette entreprise ? Quel sens prend le pèlerinage dans un âge où l'unité du monde catholique a été remise en cause par le Schisme, et est battue en brèche par le regain, au sein de l'Église, de tensions entre la tête et les membres (querelles entre pape et concile, Rome et Églises nationales, disputes entre ordres religieux, remise en cause des autorités religieuses par les Hussites et les autres mouvements dissidents) ? Enfin, la Réforme luthérienne, phase séparant traditionnellement les champs de recherche des médiévistes et des 
modernistes en Allemagne, a-t-elle lieu dans un contexte de critique des pèlerinages ou ceux-ci reçoivent-ils encore la faveur des croyants ?

Les effets de la multiplication des sources au cours des derniers siècles du Moyen Âge sont tels que l'on peut entreprendre une analyse chronologique et spatiale plus fine. On redécouvre alors qu'au-delà de Rome, Jérusalem, Compostelle, qui restent malgré tout les Big Three du pèlerinage de l'Europe occidentale, de nombreux lieux suscitent la dévotion assidue des pèlerins de tous rangs. De plus, en abordant l'espace du Saint-Empire, c'est une sorte de terra incognita historiographique que l'on dévoile. Le Saint-Empire n'est pas un espace négligeable dans l'Europe médiévale, mais reste encore peu connu pour le chercheur français en littérature ou histoire médiévale. Cet état de fait est dû à la barrière de la langue, celle des sources - puisqu'à partir du $\mathrm{XIII}^{\mathrm{e}}$ siècle une part croissante de la production se fait en langue vernaculaire -, comme celle d'une historiographie de langue allemande aussi importante que la nôtre, mais aussi à la relative indifférence mutuelle des espaces «français » et «allemands» au Moyen Âge; il faut sans doute ajouter à cela une conception française trop occidentale de l'Europe. Cependant, effet d'un volontarisme non seulement politique mais aussi scientifique $^{1}$, de nombreuses entreprises de rapprochement historiographique entre France et Allemagne ont vu le jour et jeté les bases d'une histoire croisée de ces deux espaces sur le long terme ${ }^{2}$.

C'est dans cet objectif de présentation d'une question encore ouverte, d'élargissement des horizons historiographiques au-delà du Rhin, que j'ai

\footnotetext{
${ }^{1}$ On songe aux efforts de l'Institut Historique Allemand (Paris), de l'Institut Français d'Histoire en Allemagne (Francfort-sur-le-Main), ainsi que de l'université francoallemande de Sarrebruck.

${ }^{2}$ Vient par exemple de paraître pour la fin du Moyen Âge le deuxième tome d'une Histoire franco-allemande. Cf. Jean-Marie MoEgLIN, Kaisertum und allerchristlichster König: 1214-1500, Darmstadt, Wissenschaftliche Buchgesellschaft, «Deutschfranzösische Geschichte », 2, 2010.
} 
proposé ce sujet dans le cadre du séminaire Questes. Le thème des pèlerinages ne correspond pas à mes propres travaux de recherche et mon exposé s'appuie de ce fait non sur des travaux personnels mais sur la lecture de quelques ouvrages que j'ai mis en perspective à partir des questions posées en introduction, dans le cadre d'une réflexion méthodologique. Je montrerai dans un premier temps comment on peut entreprendre l'étude des pèlerinages dans le contexte plus large de l'historiographie médiévale allemande, et en particulier en considérant le pèlerinage dans le cadre de l'histoire des mobilités. Ce point de vue permettra de faire une sorte de présentation spatiale des pèlerinages dans le Saint-Empire. Enfin, on examinera un cas relativement connu en Allemagne, celui du pèlerinage du Saint-Sang de Wilsnack, cas original d'un pèlerinage éphémère dans une région encore périphérique de l'Empire.

\section{Une histoire des mobilités}

Aborder l'espace de l'Empire par la question du pèlerinage peut se faire de deux manières. La plus évidente est de considérer l'Empire comme simple cadre géographique d'étude, espace de départ à travers lequel on tente d'analyser les particularités des pèlerins allemands, tant du point de vue social que du point de vue de leur parcours ${ }^{3}$. Moins connu comme espace de destination, l'Empire contient de nombreux lieux de pèlerinages qui attirent non seulement les pèlerins de cet espace politique, mais aussi, parfois, des pèlerins d'autres espaces. Dans les deux cas, on peut remarquer que l'historiographie des pèlerinages s'inscrit dans le contexte plus large de

\footnotetext{
${ }^{3}$ Par exemple, l'itinéraire des Allemands vers Jérusalem se différencie de l'itinéraire français en étant beaucoup plus orienté vers l'Italie; cette différence met en lumière l'importance géopolitique et économique des régions des cols alpins autour des cantons suisses et du Tyrol aux $\mathrm{XV}^{\mathrm{e}}$ et $\mathrm{XVI}^{\mathrm{e}}$ siècles, ainsi que le rôle de Venise dans les contacts entre Méditerranée et Europe du Nord.
} 
l'histoire des mobilités au Moyen Âge, qui me semble avoir retenu une attention plus grande de la part des historiens allemands. L'espace allemand apparaît au Moyen Âge comme un espace relativement mobile, et c'est tout un éventail de concepts historiographiques qui se propose à nous. Le pouvoir royal est encore une Reiseherrschaft, un «pouvoir itinérant» se déplaçant constamment le long de chemins rebattus entre les villes d'Empire et les résidences des souverains dans leurs terres patrimoniales ${ }^{4}$. Les princes et les villes d'Empire, autres maillons de l'Empire, participent à ce pouvoir décentré lors de diètes régulières ( $\mathrm{Tag}$ ), et grâce à une communication permanente qui se traduit concrètement par l'envoi de messagers et d'ambassades qui sillonnent les chemins de l'Empire. La sécurité des routes fait partie des attributions des princes depuis 1231, et apparaît comme l'un des fondements de leur souveraineté au même titre que le droit de battre monnaie ou d'établir des douanes: le Geleitrecht («droit de conduit ») est une nouvelle source de puissance, et une réalité pour quiconque voyage dans l'Empire. De même, la multiplication des universités dans l'Empire à partir de 1348 permet l'augmentation du nombre d'étudiants. Le développement des réseaux marchands appartient, lui aussi, à l'histoire des mobilités. Enfin, ce champ de recherche s'enrichit de la prise en compte des nombreux ouvrages de cartographie et de géographie du XVI ${ }^{\mathrm{e}}$ siècle, d'un Waldseemüller ou d'un Sebastian Münster par exemple.

Pour conclure sur cette histoire des mobilités, je rappellerai l'existence d'une bibliographie analytique en trois tomes des récits de voyage à la fin du Moyen Âge consacrée aux récits allemands, français et

\footnotetext{
4 Fondée en 1985, la Residenzenkommission de l'Académie des Sciences (http://resikom.adw-goettingen.gwdg.de/index.php) a publié plusieurs dizaines d'ouvrages fondamentaux sur la formation des résidences des princes de tous rangs dans la transition entre Allemagne médiévale et moderne. Ce sont des centaines de villes qui ont profité de l'implantation de ces cours, et leur étude ressortit à la fois de l'histoire urbaine et de l'histoire palatiale (Pfälzenforschung).
} 
néerlandais ${ }^{5}$; ce travail, dirigé par Werner Paravicini, a été numérisé et est désormais essentiel pour quiconque veut étudier l'histoire des voyages à cette époque. L'analyse de certains récits montre que le pèlerinage n'est pas forcément le but principal d'un voyage. Si on ne peut exclure le sens religieux de la pérégrination, il reste que parfois le voyage vers un lieu saint est un simple élément, presque touristique, d'un itinéraire poursuivant un but plus immédiat. Pour certains princes allemands de la fin du Moyen Âge, le voyage à Jérusalem s'apparente à une mode, un Grand Tour avant la lettre, le Saint-Sépulcre servant parfois de lieu d'adoubement ${ }^{6}$.

\section{Les pèlerinages dans l'Empire : présentation sommaire}

La bibliographie allemande sur les pèlerinages est souvent peu satisfaisante. Le sujet, populaire s'il en est parmi les thèmes médiévaux, fait qu'une partie importante de la production s'apparente à de la vulgarisation scientifique, laquelle, pour être correcte sur le fond, n'apporte pas forcément de contenu original. Par ailleurs, on peut déceler deux tentations marginales qui risquent de rendre anhistorique l'étude des pèlerinages : d'une part, une sorte de psychologie du pèlerinage, tâchant par exemple d'interpréter trop précisément les raisons du pèlerinage (la recherche de l'inconnu, la recherche du pardon, l'expiation d'une faute) et, d'autre part, la tentation de faire de la Volkskunde - terme qu'on ne peut qu'imparfaitement traduire par « ethnologie »-, qui le plus souvent quitte les bornes historiques pour aborder une sorte d'analyse figée des rituels de pèlerins. Enfin, le cadre géographique de l'Empire comme espace de pèlerinages ne va pas de soi dans l'historiographie. Il faut donc, la plupart

5 Europäische Reiseberichte des späten Mittelalters. Eine analytische Bibliographie, Werner PARAVICINI (dir.), Francfort-sur-le-Main, P. Lang, 1994-2000, 3 vol., http://www.digiberichte.de.

${ }^{6}$ Cf. Cordula NOLTE, «Erlebnis und Erinnerung: Fürstliche Pilgerfahrten im 15. Jahrhundert », in Irene ERFEN et Karl-Heinz SPIESS (dir.), Fremdheit und Reisen im Mittelalter, Stuttgart, Steiner, 1997, p. 65-92. 
du temps, se confronter ou bien à une histoire générale européenne des pèlerinages, ou bien à des monographies locales ou régionales qui ne permettent qu'imparfaitement d'opérer des comparaisons quantitatives et qualitatives entre les différents pèlerinages.

Ce qui ressort toutefois de cette bibliographie est la multiplicité des lieux de pèlerinage. La faiblesse des sources quantitatives ou l'absence d'études précises, cependant, rend délicate toute tentative de hiérarchisation. Parfois, on ne peut réussir à expliquer la plus grande popularité d'un lieu de pèlerinage par rapport à un autre. En Alsace, on peut trouver à quelques lieues de distance le pèlerinage de Saint-Thiébaut de Thann ${ }^{7}$, dans une ville sous suzeraineté Habsbourg, pèlerinage dont on possède quelques récits de miracles mentionnant des pèlerins du Nord de l'Allemagne, et celui de Dusenbach ${ }^{8}$, dans les terres des seigneurs de Ribeaupierre; le premier semble avoir une importance suprarégionale, le second davantage régionale.

Une des difficultés de l'Empire était le manque de saints pouvant se rattacher directement aux temps des Apôtres et aux premiers siècles de l'Église, mis à part la figure de l'évangélisateur saint Materne de Cologne, disciple de Pierre. Progressivement cependant, l'Empire se dote de lieux saints abritant des reliques diverses. La ville de Cologne, une des premières villes de l'Empire sur tous les plans (historique, démographique, religieux et commercial), est particulièrement privilégiée : c'est la ville de Materne, le lieu de supplice de sainte Ursule et de ses onze mille vierges, et la dépositaire des reliques des Rois Mages. Frédéric $\mathrm{I}^{\mathrm{er}}$ Barberousse en a fait don à son chancelier, l'archevêque Rainald de Dassel, après leur découverte providentielle près des murailles de la ville rebelle de Milan en 1165. Non

\footnotetext{
${ }^{7}$ Cf. Tomus miraculorum sancti Theobaldi, Georg STOFfel (éd.), Colmar, Jung, 1875.

${ }^{8} \mathrm{Cf}$. Francis RAPP, «Le pélerinage de Dusenbach et Maximin II de Ribeaupierre », Revue d'Alsace, 128 (2002), p. 193-203.
} 
loin de là, Aix-la-Chapelle abrite le buste-reliquaire de Charlemagne, cadeau de son lointain successeur Charles IV. En Suisse, l'abbaye d'Einsiedeln, lieu central du duché de Souabe dès le IX ${ }^{\mathrm{e}}$ siècle $^{9}$, favorise le développement d'un important pèlerinage marial. Sa situation contribue à sa popularité : elle est proche du col du Saint-Gothard, une des routes principales permettant de traverser les Alpes centrales à partir des années 1220 et qui servira le désenclavement de la Suisse. C'est là que le Thuringien Hermann Künig von Vach commence son itinéraire vers Compostelle en 1495. Il n'est pas non plus anodin de voir que le futur réformateur de Zurich, Ulrich Zwingli, y a été prédicateur, avant de s'opposer au trafic d'indulgences. De nombreux autres lieux de pèlerinages peuvent être cités, notamment Saint-Nicolas-de-Port, cœur religieux de la Lorraine ducale, lieu de foire pour les marchands sur la route du sel lorrain, Saint-Thiébaut de Thann se trouvant justement sur cette route, mais de l'autre côté des Vosges.

On peut aussi observer la naissance tardive de certains pèlerinages : le culte de la sainte tunique du Christ, à Trèves, semble naître sous Maximilien, bénéficiant de la diffusion de huit ouvrages imprimés en 1512 et $1513^{10}$. De même, un miracle attribué à la Vierge lors de la destruction des bâtiments des Juifs de Ratisbonne (après la mort de leur protecteur Maximilien en 1519), provoque l'émergence rapide du principal pèlerinage de Bavière autour d'une statue de Madone sculptée l'année suivante. Le passage à la Réforme en 1542 provoque toutefois l'arrêt du pèlerinage pour deux siècles : à la place du lieu de vénération sera érigée la première église protestante de la ville. Ainsi un pèlerinage peut fleurir au temps de Luther,

\footnotetext{
${ }^{9}$ Cf. Helmut MAURER, Der Herzog von Schwaben : Grundlagen, Wirkungen und Wesen seiner Herrschaft in ottonischer, salischer und staufischer Zeit, Sigmaringen, Thorbecke, 1978.

${ }^{10}$ Cf. Der Heilige Rock zu Trier. Studien zur Geschichte und Verehrung der Tunika Christi, Erich ARETZ, Michael EMBACH, Martin PERSCH et Franz Ronig (dir.), Trèves, Paulinus-Verlag, 1996.
} 
et ce avant même qu'on puisse parler de Contre-Réforme.

Une tentative de cartographie des principaux lieux de pèlerinages montre assez aisément leur concentration dans les zones méridionales (Alpes) et occidentales (Rhin) de l'Empire. C'est, en s'autorisant quelques approximations, la zone de l'ancien limes et des premiers contacts avec le monde romain; c'est, pendant de nombreux siècles, le lieu principal d'implantation du pouvoir impérial, la partie la plus urbanisée de l'Empire. Il faudra en effet attendre les XII ${ }^{\mathrm{e}}$ et XIII ${ }^{\mathrm{e}}$ siècles pour que soient vraiment rattachées à la sphère culturelle de l'Empire les régions du Nord et de l'Est, et que se développe le chapelet urbain de l'espace hanséatique. Il reste que ces «nouvelles régions» de l'Empire présentent une densité démographique beaucoup plus faible et un maillage urbain moins serré que dans l'Ouest. Il est assez naturel que, de ce fait, le nombre de lieux saints et leur importance aient été moindres.

Mais cette zone occidentale et méridionale correspond aussi aux itinéraires politiques et économiques majeurs de l'Empire. On a vu plus haut la situation de Saint-Nicolas-de-Port et de Saint-Thiébaut de Thann sur la route entre Italie et Flandre qui traverse l'Alsace, la Lorraine et la Champagne. Les relations avec l'Italie, et particulièrement avec Rome, provoquent le franchissement régulier des passages alpins par les troupes impériales et les marchands. Naturellement, les lieux de pèlerinages rhénans et alpins peuvent aussi faire partie de l'itinéraire vers Compostelle, Rome ou Jérusalem, se rattachant par là au grand réseau européen religieux.

Il est, de ce fait, intéressant d'analyser un cas particulier qui ne corresponde pas à cette situation majoritaire des grands lieux de pèlerinage, celui de Wilsnack; et ce d'autant plus que les péripéties autour des hosties ensanglantées font mieux ressortir les enjeux politiques, financiers et théologiques d'un pèlerinage à la fin du Moyen Âge dans l'Empire. 


\section{Wilsnack : naissance et mort d'un pèlerinage}

Les différentes phases de la vie de Wilsnack sont largement tributaires du contexte politique et religieux des $\mathrm{XIV}^{\mathrm{e}}, \mathrm{XV}^{\mathrm{e}}$ et $\mathrm{XVI}^{\mathrm{e}}$ siècles : à son origine, la formation éphémère d'un espace politique de grande ampleur, et à sa fin, l'établissement de la Réforme dans la région. Entre-temps, le pèlerinage a été l'objet de longs débats, que Hartmut Boockmann a pu analyser comme un cas emblématique des tentatives de réforme de l'Église au quinzième siècle ${ }^{11}$.

Wilsnack (aujourd'hui Bad Wilsnack) est, au XIV siècle, un village de la Prignitz, petit pays au Nord-Ouest de la marche de Brandebourg, dans le diocèse de Havelberg. Effet de la conquête chrétienne de ces régions encore païennes au début du XII ${ }^{\mathrm{e}}$ siècle, la Prignitz est plus ou moins sous le contrôle des margraves de Brandebourg, même si de nombreux petits seigneurs, ainsi que les évêques alentour (celui de Havelberg, et le puissant archevêque de Magdebourg) possèdent aussi des parcelles d'autorité et n'hésitent pas à entreprendre des actions guerrières personnelles. Après un raid d'un de ces seigneurs en 1383, le curé de Wilsnack, Johannes Cabuez, découvre au milieu des ruines de l'église trois hosties ayant pris la couleur du sang. À partir de cette découverte miraculeuse, tout un processus s'enclenche, faisant en peu de temps de Wilsnack un lieu majeur de pèlerinage pour toute l'Europe du Nord.

De nombreux facteurs peuvent expliquer ce succès. Tout d'abord, on semble avoir eu, en Europe du Nord et de l'Est, une certaine vénération pour des miracles liés au sang du Christ : Wilsnack n'est qu'un cas parmi

11 Hartmut BoockmanN, «Der Streit um das Wilsnacker Blut. Zur Situation des deutschen Klerus in der Mitte des 15. Jahrhunderts », Zeitschrift für historische Forschung, 9 (1982), p. 385-408. 
d'autres ${ }^{12}$. De manière pragmatique, il n'est pas besoin de reliques de saints pour susciter l'admiration des foules: une hostie ou une représentation picturale peuvent suffire pour créer le miracle. Miracle fabriqué par le curé, ou couleur due à une bactérie aux effets désormais connus (bacterium prodigiosum $)^{13}$, les deux explications restent possibles. Les détracteurs du miracle accuseront les Franciscains de Magdebourg d'avoir fabriqué un miracle similaire pour pouvoir financer la construction d'une nouvelle église.

On peut cependant expliquer ce succès en abordant une perspective géopolitique qui puisse expliquer pourquoi, en ce lieu et à ce moment-là, apparaît un pèlerinage. Tout d'abord, depuis 1373, le Brandebourg est aux mains d'une seule famille, qui possède aussi le royaume de Bohême, la Silésie et les Lusaces: les Luxembourg, depuis le début du siècle, ont réussi, malgré les partages inévitables entre cousins, à créer un espace patrimonial considérable et continu. De plus, cette famille fournit quatre empereurs de 1348 à 1437, avec une seule interruption; et Sigismond, le dernier d'entre eux, est le maitre du grand royaume de Hongrie. C'est une période où la moitié orientale de l'Europe opère un rattrapage politique, économique et démographique sur la moitié occidentale plus développée. Non seulement la Bohême, mais aussi la Pologne et la Hongrie sont désormais des États pérennes, après quelques siècles d'incertitudes. Les espaces de la Mer Baltique sont dorénavant partie prenante du système économique européen, avec tout un réseau de villes germanophones littorales qui exportent vers l'Ouest les produits de l'intérieur des terres. Bientôt, la Pologne deviendra le grenier à blé de l'Occident, une fois

12 Cf. Johannes HeuSER, "Heilig-Blut» in Kult und Brauchtum des deutschen Kulturraumes. Ein Beitrag zur religiösen Volkskunde, Diss. Phil., Bonn, 1948.

${ }^{13}$ Cf. Alois DörING, «Bluthostien », in Michael BUCHBERGER, Walter KASPER et Konrad BAUMGARTNER (éd.), Lexikon für Theologie und Kirche, Fribourg-en-Brisgau, Herder, 2006, vol. 2, col. 539. 
Dantzig reprise aux Teutoniques. Le miracle du Sang intervient dans une région qui s'intègre désormais au reste de l'Europe occidentale, le long de routes commerciales continentales qui vont notamment favoriser l'émergence de Berlin comme ville principale de la région.

Le rôle du pouvoir est évident si l'on analyse les débuts de Wilsnack. Dès le miracle connu, le pape et les différents évêques de la région proclament des indulgences, ce qui contribue à la popularité du lieu ; mais des Luxembourg aussi s'y rendent. Les Hohenzollern, qui obtiennent la marche de Brandebourg sous Sigismond, continueront à faire de Wilsnack leur pèlerinage familial.

La recherche sur Wilsnack n'est pas encore complète. On n'a pas pu analyser l'impact du pèlerinage sur toutes les régions autour de Wilsnack ${ }^{14}$. Si peu de Polonais ont fait le voyage (très certainement parce qu'il y avait des pèlerinages concurrents), Wilsnack apparaît en revanche comme un lieu privilégié pour tous les Allemands du Nord, attirant de même de nombreux Anglais et Scandinaves. Il semble y avoir eu plusieurs phases de développement du pèlerinage, avec des périodes de crises qui correspondent souvent à des tensions au sein de l'Église : la remise en cause de ces miracles par le théologien de Bohême Jan Hus, puis les guerres menées par les Hussites qui ravagent la région de 1410 à 1434 sont l'occasion d'une pause, avant un regain de popularité dans la seconde moitié du $\mathrm{XV}^{\mathrm{e}}$ siècle. Quoi qu'il en soit, le succès du pèlerinage profite à l'ensemble de la communauté. On finit par construire une grande halle, une plus grande église capable d'accueillir davantage de pèlerins (de même, le nombre d'auberges ne suffit pas), et Wilsnack obtient le statut de ville au tournant du $\mathrm{XVI}^{\mathrm{e}}$ siècle.

${ }^{14}$ Cf. Die Wilsnackfahrt: Ein Wallfahrts- und Kommunikationszentrum Nord- und Mitteleuropas im Spätmittelalter, Felix ESCHER et Hartmut KÜHNE (dir), Francfort-surle-Main, Peter Lang, « Europäische Wallfahrtsstudien », 2, 2006. 
Le développement de la ville et du pèlerinage sera cependant contrecarré par les trois grandes phases de tensions réformatrices au sein de l'Église à la fin du Moyen Âge: les critiques de Hus au début du $X^{\mathrm{e}}$ siècle, les tensions entre les conciliaristes ${ }^{15}$ et les partisans du pape au milieu du siècle, et le rejet définitif des pèlerinages par Luther dans son ouvrage À la noblesse chrétienne de la nation allemande en 1520.

Dans son article ${ }^{16}$, Hartmut Boockmann met en lumière une sorte de pourfendeur des mauvais pèlerinages, le chanoine Heinrich Tocke, qui sera présenté par des historiens protestants tels Flacius Illyricus comme un préluthérien acerrimus superstitionum hostis, «ennemi implacable des superstitions $»^{17}$. Après des études à l'université d'Erfurt (celle où ira Luther plus tard), il est chanoine à Magdebourg, et fait partie des tenants de la réforme de l'Église au concile de Bâle, aux côtés de Nicolas de Cues. Il semble attendre 1443 et la visite de l'évêque de Havelberg à Magdebourg pour exprimer ses doutes anciens sur la validité du miracle, et obtient de l'évêque une enquête. Celle-ci permet de rassembler cent cinquante expertises de théologiens d'horizons divers, dont une majorité cependant conteste la véracité du miracle ou la légalité du pèlerinage. Le nombre de traités théologiques est à la mesure de l'importance du pèlerinage, et des inquiétudes doctrinales.

En effet, si Tocke ne peut prouver que le miracle soit fabriqué, il montre en revanche le péril dans lequel semble être tombé le peuple : l'adoration non pas de l'action divine, mais de l'objet du miracle, ces hosties qui ont d'ailleurs fini par se détériorer dans leur reliquaire, et qui, peut-être, n'ont même pas été consacrées. C'est ce dont témoigne le

\footnotetext{
${ }^{15}$ Les conciliaristes remettaient en cause l'autorité supérieure du pape sur les conciles œcuméniques, en conséquence notamment du Grand Schisme d'Occident (1378-1417) et des conciles qui y avaient mis fin.

${ }^{16}$ Hartmut BoockMANN, « Der Streit um das Wilsnacker Blut... », art. cit., p. 391 sqq.

${ }^{17}$ Mathias Flacius Illyricus, Catalogus testium veritatis, qui ante nostram aetatem reclamarunt Papae, Bâle, 1556, p. 966.
} 
Chartreux Johannes Hagen lorsqu'il vient à Wilsnack pour vérifier de ses propres yeux : «Ac diligentissime perspexi, ac dixi : "Quid nunc populus adorat ?" ». On lui montre les hosties de sang: « Has particulas ». Hagen donne son diagnostic : «Nunc horrui. Ecce : manifesta idolatria ${ }^{18}$.

Tout le monde n'est cependant pas de l'avis de Tocke. Par exemple, Mathias Döring est favorable au pèlerinage à plusieurs titres : tout d'abord en tant que général des Franciscains, ordre prompt à reconnaître la validité des miracles du Sang (contrairement aux Dominicains, plus critiques); ensuite en tant que sujet de l'Électeur de Brandebourg, l'un des principaux bénéficiaires du pèlerinage avec l'évêque de Havelberg; et enfin parce que l'Électeur a la faveur du pape, et a choisi le camp de ce dernier contre celui du concile.

La situation n'est pas simple, et la ligne de fracture entre conciliaristes et partisans du pape ne recoupe pas totalement les oppositions entre Franciscains et Dominicains, et entre pro- et anti-Wilsnack. Heinrich Tocke réussit cependant à convaincre son collègue conciliariste Nicolas de Cues, légat pontifical en Allemagne, d'interdire la vénération des hosties ensanglantées en 1451. La crise éclate, avec l'excommunication mutuelle des évêques de Havelberg et de Magdebourg, suivie d'entreprises militaires entre Brandebourg et Magdebourg. Le pape, en dernier recours, fait le choix de contenter l'Électeur de Brandebourg et casse la décision de son légat.

Wilsnack survit à la crise réformatrice du milieu du siècle. Le pèlerinage ne peut cependant que disparaître avec l'implantation progressive de la Réforme, tolérée par les Électeurs de Brandebourg à partir

\footnotetext{
18 «Alors j'ai examiné avec attention, et j'ai demandé: "Mais qu'adore à présent le peuple ?" -“Celles-ci". Alors je fus pris d'horreur. Voilà une idolâtrie manifeste » (Hartmut BoocKmANN, «Der Streit um das Wilsnacker Blut... », art. cit., p. 392. Nous traduisons). Cf. Josef KLAPPER, Der Erfurter Kartäuser Johannes Hagen. Ein Reformtheologe des 15. Jahrhunderts, Leipzig, St. Benno-Verlag, «Erfurter theologische Studien », 9-10, 1961, vol. 2, p. 105.
} 
de 1535. À la mort de l'évêque de Havelberg, la communauté de Wilsnack élit un pasteur, Joachim Ellefeldt. Un bras de fer s'engage entre Ellefeldt et le doyen d'Havelberg, et en 1552 le pasteur décide de détruire les hosties et leur reliquaire, symbole même du pèlerinage. Le sacrilège est déposé et banni, ce qui est une sorte de compromis entre le châtiment sévère et l'indulgence. Si ce coup ne met pas fin immédiatement au pèlerinage, et on le comprend puisque cet attentat n'est pas condamné explicitement par des autorités mais est l'effet de l'audace d'une partie de la communauté, le pèlerinage de Wilsnack périclite rapidement.

Le pèlerinage de Berlin à Wilsnack semble renaître depuis quelques années, mais il est fort probable que cela participe du processus plus ou moins conscient de construction d'une identité historique du Land de Brandebourg qui vit mal son statut de Land «autour de Berlin »- sa capitale naturelle -, et l'association avec la Prusse, entité politique qui n'a englobé le Brandebourg que pendant trois siècles ${ }^{19}$. Wilsnack serait un de ces lieux historiques qui permettraient au Brandebourg de créer une identité dont elle poursuit, peut-être vainement, la quête.

En conclusion, le cas de Wilsnack montre que l'on ne peut résumer l'histoire des pèlerinages à l'image des croisés de Jérusalem, des pèlerins du camino francés et des visiteurs de Rome. Pendant quelques décennies, un lieu qui était deux siècles auparavant aux confins de la Chrétienté a attiré des milliers de pèlerins, contribuant à renverser partiellement les itinéraires de pèlerinages allant traditionnellement du Nord vers le Sud et de l'Est vers l'Ouest. La question des rapports entre pèlerinages et Réforme reste posée, et renvoie aux débats plus généraux sur les facteurs de

\footnotetext{
${ }^{19}$ L'université de Potsdam semble en tout cas avoir pris clairement conscience du rôle qu'elle a à jouer, en tant que Landesuniversität fondée en 1991, dans la redécouverte de l'histoire du Brandebourg et dans la mise en valeur de ses lieux historiques inconnus du grand public.
} 
développement de la Réforme ou de maintien strict du catholicisme selon les régions. Si certains pèlerinages sont suffisamment importants pour attirer sans peine les pèlerins de toutes nations, on observe ainsi, à l'exemple des pèlerinages d'Empire, une certaine hiérarchisation qui fait de certains des lieux viables, et d'autres des lieux fragiles. Très souvent, en plus des enjeux de dévotion, perdurent des enjeux politiques et économiques de contrôle entre, par exemple, le souverain et l'autorité ecclésiastique locale. Le pèlerinage est abandonné à son sort lorsque le souverain décide de mettre fin au conflit avec cette autorité ecclésiastique pour créer une autre Église. 